\title{
La dissociation triple : le statut d'une preuve dans le domaine de la neurobiologie de la mémoire
}

\section{Françoise Schenk}

\section{OpenEdition}

\section{Journals}

Édition électronique

URL : http://journals.openedition.org/ress/414

DOI : 10.4000/ress.414

ISSN : 1663-4446

Éditeur

Librairie Droz

Édition imprimée

Date de publication : 10 décembre 2003

Pagination : 161-171

ISBN : 2-600-00913-2

ISSN : 0048-8046

Référence électronique

Françoise Schenk, "La dissociation triple : le statut d'une preuve dans le domaine de la neurobiologie de la mémoire », Revue européenne des sciences sociales [En ligne], XLI-128 | 2003, mis en ligne le 11 novembre 2009, consulté le 19 avril 2019. URL : http://journals.openedition.org/ress/414 ; DOI : $10.4000 /$ ress.414 
Françoise SCHENK

\section{LA DISSOCIATION TRIPLE: LE STATUT D'UNE PREUVE DANS LE DOMAINE DE LA NEUROBIOLOGIE DE LA MÉMOIRE}

SOMMAIRE: 1. Particularités de la recherche en psychobiologie. - 2. Multiplicité des formes et des systèmes de mémoire. - 3. La dissociation triple. 4. Après la preuve. -5 . Conclusion.

La publication en 1993 d'un article intitulé «A triple dissociation of memory systems: hippocampus, amygdala, and dorsal striatum» par R. McDonald et N. White a marqué une étape importante dans le domaine de la neurobiologie de la mémoire. Il offrait, sur la base d'un schéma expérimental élégant, la preuve d'une indépendance entre trois formes de mémoire complémentaires et leurs soubassements neuronaux. Cependant, ce travail n'a pas eu un retentissement exceptionnel et n'a pas donné lieu à une publication dans Nature ou Science, ce que l'on attendrait d'un résultat expérimental très marquant. Il y a plusieurs raisons à cette indifférence relative, certaines inhérentes aux déterminants sociaux de l'activité de recherche, d'autres liées au statut particulier d'une démonstration formelle dans ce domaine, donc plus épistémologiques. Comme nous le verrons, «la preuve» vient plutôt clore et resserrer un ensemble de connaissances, sans rien amener de réellement nouveau. Les données qui font l'avancement de la connaissance s'accumulent le plus souvent à l'allure de lentes érosions qui viennent miner des résistances cachées. Ce processus est entrecoupé parfois de propositions audacieuses, nées d'un coup de génie créateur, dont le caractère de vérité scientifique n'est établi que bien plus tard, une fois que tous les contours de cette nouvelle figure ont été redessinés dans une approche plus impressionniste que rigoureuse. C'est alors qu'une preuve au sens formel du terme peut venir marquer son empreinte, comme une sorte de sceau sur une charte.

Les progrès dans le domaine de la neurophysiologie de la mémoire au cours de la seconde moitié du XX $\mathrm{XX}^{\mathrm{e}}$ siècle ont trois faces constituées d'autant de résistances ou de difficultés à surmonter. Tout comme pour la découverte de la circulation du sang par Harvey, il fallait faire une sorte de saut épistémologique pour dépasser un consensus fondé sur le bon sens ${ }^{1}$.

Tout d'abord, le constat de la multiplicité des formes de mémoire devait passer par la reconnaissance du fait que les amnésiques graves enregistraient des traces multiples de leur vécu quotidien alors qu'ils étaient manifestement figés dans

Puisque les vaisseaux capillaires étaient invisibles, la circulation du sang ne pouvait donc se faire «à circuit fermé » et le sang quittant les artérioles devait immerger les tissus avant de reparaître dans les veinules. 
l'instant présent. Comme ces traces n'étaient pas conscientes, elles ne prêtaient pas à l'affirmation «Je me souviens », la seule signature acceptée pour témoigner d'une mémoire de type humain, consciente et accessible à l'introspection directe.

Le second blocage tient au fait que les données cliniques désignaient systématiquement les dommages d'une structure de la profondeur du lobe temporal comme responsables des amnésies graves. Or cette structure est au cœur de ce qui a été appelé le système limbique, vu initialement comme un témoignage archaïque du cerveau animal, donc peu susceptible de jouer un rôle clé dans la mémoire humaine.

Le troisième frein est lié au fait que l'approche expérimentale ne pouvait être menée que chez l'animal, très vraisemblablement dépourvu de mémoire épisodique digne de ce nom. Pour résumer la position des chercheurs dans les années 1960, l'animal apprenait sans se souvenir alors que l'Homme se souvenait sans avoir besoin d'apprendre. Il fallait donc développer les théories du comportement animal pour y trouver des formes de mémoires qui aient des propriétés semblables à celles de la mémoire humaine... sans prendre le langage pour attester de cette mémoire.

Ces trois biais ont donc considérablement ralenti la recherche puisqu'ils n'étaient pas reconnus comme tels par la communauté scientifique dans sa majorité et rendaient difficilement acceptables des résultats expérimentaux qui tendaient à les contredire. Des présupposés très semblables ont ralenti les progrès de ce que l'on nomme les théories de l'esprit qui examinent dans quelle mesure des animaux peuvent décoder les intentions d'un partenaire (Gallese \& Goldman, 1998).

Nous allons montrer dans l'histoire récente de la mémoire, comment une proposition provocante, suivie d'une maturation basée sur des séries d'expériences plus ou moins contradictoires, a progressivement mené à une sorte de consensus expérimental et théorique à l'origine de l'expérience de Mc Donald et White, lui donnant ainsi un statut de preuve rassemblant tout le travail qui s'est fait en amont, sur une trentaine d'années. Cette clôture annonce et permet de nouvelles recherches, de nouvelles remises en question. Comme un resserrement dans le lit d'un fleuve rassemblerait les vecteurs de force avant de les laisser rejaillir à l'occasion d'un élargissement ultérieur. Ce qui explique peut-être le retentissement modéré de ce travail dans l'immédiat en dépit de son caractère déterminant.

\section{PARTICULARITÉS DE LA RECHERCHE EN PSYCHOBIOLOGIE}

La difficulté majeure de la psychobiologie tient à la diversité des niveaux d'explication qu'elle se doit d'intégrer. Psychologie, elle part de descriptions du comportement selon des théories qui en ignorent les déterminants biologiques. Biologie, elle traite des organismes, des organes, des systèmes, des cellules et des réactions chimiques. Avec le risque de croire que le niveau le plus «bas», celui des réactions entre molécules, pourrait être le plus simple. Cette simplicité apparente tient au fait qu'il est facile, techniquement, de se concentrer sur une réaction sans avoir besoin de tenir compte de l'ensemble des facteurs susceptibles d'en influencer le déroulement, dans un réductionnisme pourtant fructueux. 


\section{- La neurobiologie et ses niveaux d'explication}

Pour utiliser une métaphore, on pourrait dire que la neurobiologie aborde la mémoire comme s'il s'agissait d'une tapisserie ancienne et précieuse, récemment découverte. Elle y entrevoit une scène complexe, qui pourrait être l'assemblage d'épisodes vécus, dans toutes leurs répercussions sensorielles et motrices sur l'individu qui en a été le centre. Elle se préoccupe des principes qui ont été à l'origine de la juxtaposition d'éléments très divers dans cette scène, images, odeurs, mouvements, émotions, humeurs, contrastes. Elle cherche à intégrer ces mécanismes dans une description biologique du fonctionnement cérébral, selon une démarche matérialiste et réductionniste. Bien plus que de la scène elle-même, le neurobiologiste se préoccupe de la structure des motifs, de leur composition, de leurs relations ainsi que de la trame dans laquelle ils s'inscrivent. Il veut surtout comprendre les points utilisés, les mécanismes de production, la composition des fils, des teintures, selon le réductionnisme qui le caractérise.

Cette décomposition en niveaux de plus en plus éloignés de la réalité vécue veut pourtant fonder des interventions et des projets de restauration plus globaux de la tapisserie: consolider les fils fragilisés, activer des processus permettant de résister à l'usure, aux insectes et aux intempéries ${ }^{2}$. Et comme la mémoire vivante est en devenir, il faut encore s'assurer que la tapisserie pourra continuer sa croissance et son développement, dans une cohérence qui respecte celle des expériences de l'individu. Avec un présupposé peut-être provocateur: la mémoire n'est pas un instrument visant à recomposer le passé, mais à compléter le présent par des informations permettant une adaptation optimale : la porte arrière de la maison pourrait offrir une issue de secours, cette lueur dans le regard de mon père évoque une fessée, le rayon de soleil qui éclaire ce meuble familier est forcément matinal.

\section{- La notion de systèmes pour résister au réductionnisme ultime}

On voit immédiatement dans ce qui précède la difficulté d'une démarche qui se centre sur des mécanismes élémentaires, tout en affirmant sa pertinence dans le cas de la mémoire humaine. Comment s'assurer qu'aucune propriété fondamentale de la mémoire n'est perdue quand on l'aborde comme une somme de réactions intra- et intercellulaires, bref quand ses dimensions sociales et psychologiques sont abandonnées au profit d'une lecture physico-chimique?

En réalité, ce procès est lui aussi réductionniste, car il ne tient pas compte de la diversité des facettes de la neurobiologie. Celle-ci se fonde sur l'approche systémique qui a fait la force de la physiologie depuis qu'elle s'est séparée de l'anatomie pour devenir une «anatomie en mouvement», inscrivant les unités fonctionnelles dans des systèmes de relations et de répercussions multiples, pour assurer des fonctions comme l'épuration et la circulation du sang, par exemple. La physiologie reste à la base de l'essentiel des explications du vivant qui veulent rendre compte d'un fonctionnement d'ensemble, fondé sur des relations entre parties. Cette discipline peut paraître dépassée actuellement, étant donné la préférence pour une biologie moléculaire ou génétique, ou pour les «neurosciences", vaste amalgame à la mode. La physiologie peut rendre compte de l'ensemble des

2 ... à savoir, le vieillissement normal, les maladies et les épisodes de stress intense. 
relations qui sous-tendent une fonction, même lorsqu'elle cherche des éléments de causalité dans des signaux moléculaires (hormone, enzyme, gène).

Le système nerveux est considéré comme un ensemble de nœuds et de relations dont les interruptions sont responsables de la perte d'une fonction plus ou moins concrète (motricité, vision, parole) ou symbolique (compréhension du langage, mémoire, planification). Ces relations sont décrites comme des réactions qui ont lieu à des niveaux plus «simples », bio-physique, chimique, électrique, et s'inscrivent dans des niveaux organiques et de populations (de cellules, d'organes ou d'individus). Avec encore une dimension supplémentaire qui est celle de l'évolution et de l'ontogenèse.

\section{- Le dualisme à l'épreuve de la clinique}

Sans prétendre aborder directement cette question fondamentale, il faut admettre qu'elle constitue la difficulté suprême de la neurobiologie qui cherche à mettre en relation le fonctionnement cérébral et le fonctionnement mental. $\mathrm{Ne}$ serait-ce que parce que la description des fonctions mentales repose sur un découpage déterminé par les fonctions psychologiques qui n'est pas directement superposable au découpage du cerveau en structures et en systèmes (Schenk, 1992). Ce qui limite sévèrement les ambitions réductionnistes fortes du type de celles que défend Patricia Churchland (1988).

La clinique révèle un ensemble d'altérations et de dissociations fonctionnelles qui ont formé la base de découpages et de regroupements de fonctions, mettant en évidence des complémentarités, des indépendances et des relations hiérarchiques entre capacités mentales préservées ou détériorées selon les cas. Et comme les altérations observées résultent généralement de souffrances cérébrales localisées, les unités de fonctions mentales, perdues ou préservées, et révélées par ces décapages pathologiques, ont été en quelque sorte associées aux endroits de souffrance systématique selon une topologie fonctionnelle qui facilite les interprétations. C'est la lecture topologique que l'on retrouve dans un grand nombre de représentations du fonctionnement physiologique, cérébral ou somatique, dans la médecine occidentale ou orientale. Pour exemple les méridiens et les chakras des médecines traditionnelles orientales qui expriment eux aussi ce besoin de spatialiser la distribution des fonctions et dont on trouve les limites caricaturales dans la phrénologie de Gall.

Ainsi, en plus du constat un peu brutal, «no brain, no mind», la clinique a permis un redécoupage dans l'analyse d'unités fonctionnelles mentales et de leurs relations avec des systèmes cérébraux.

\section{MULTIPLICITÉ DES FORMES ET DES SYSTÈMES DE MÉMOIRE}

Avant de pouvoir énoncer l'hypothèse de trois systèmes de mémoire, animés par trois systèmes cérébraux distincts, à l'œuvre chez les mammifères en général, il a fallu formuler les propriétés de chacune de ces mémoires, les attribuer à des systèmes cérébraux distincts et accepter le présupposé que des animaux comme les rats de laboratoire expriment ces compétences d'une manière objectivable. 


\section{- Apprendre sans se souvenir : savoir faire et savoir être}

La neuropsychologie de la mémoire a été confrontée très tôt au fait qu'une amnésie même très grave ne se caractérise pas par une absence totale de traces du vécu quotidien, mais par le fait que ces traces semblent se déposer puis se manifester à l'insu du patient lui-même. Il est très vite apparu à Scoville et Milner (Milner, 1965) que même un patient aussi gravement amnésique que $\mathrm{HM}^{3}$ gardait la trace d'événements vécus sous une forme dite implicite. Alors que son comportement en offrait un témoignage flagrant, il n'avait aucun souvenir d'avoir appris à écrire en miroir, à identifier certaines images brouillées ou d'avoir entendu certains mots. Tout se passait comme si son comportement était modifié de manière durable par les circonstances, sans qu'il puisse faire référence au contexte dans lequel ces événements s'étaient produits. Il n'en gardait pas de mémoire déclaratoire, selon la définition devenue célèbre de Squire (Squire \& ZolaMorgan, 1988), il avait perdu la capacité de mémoriser un ensemble d'événements en les classant en épisodes structurés et reliés à d'autres épisodes précédents ou suivants dans une trame spatio-temporelle. Cette compréhension de l'amnésie souligne une propriété essentielle de la mémoire, qui émerge d'un processus de codage des relations spatio-temporelles entre événements. Cette propriété serait indépendante des processus par lesquels l'individu inscrirait des savoir-faire, manifestant des réponses conditionnées à des signaux particuliers, et développerait des sortes de savoir-être, sous la forme de réponses émotionnelles simples ou complexes.

Ces mémoires sont évidemment intégrées les unes aux autres dans un fonctionnement dit normal, mais elles sont capables d'autonomie. Dans les dernières pages du premier chapitre du premier volume de la Recherche du Temps Perdu ${ }^{4}$ on trouve la description de ces différentes facettes de la mémoire, sensorielle, puis émotionnelle diffuse, puis, à partir d'un point de fixation devenu explicite, capable de déplier «tout Combray dans ma tasse de thé». Pour notre propos, ce qui ressort si bien de ce texte, c'est la dissociation temporelle d'étapes d'activation de «la» mémoire: même si elles semblent s'activer les unes les autres, elles gardent une certaine indépendance. Qui n'a jamais vécu une réactivation émotionnelle intense sans cependant pouvoir accéder à une remémoration consciente du contexte auquel était attachée cette émotion?

\section{- Un découpage en trois volets}

Pour notre propos, nous retiendrons trois formes de mémoire. La première forme constitue une propriété fondamentale de la mémoire épisodique, la seconde fait la base des automatismes moteurs, la troisième réactive l'ensemble de réponses émotionnelles exprimées dans le passé en réponse à un tel stimulus 5 .

Un patient traité par la neurochirurgie en 1964 (XX?) pour réduire la sévérité de graves crises d'épilepsie et qui s'est retrouvé en même temps gravement amnésique, oubliant au fur et à mesure tous les événements, graves ou anodins de son existence.

4 Proust, M. (1924). A la recherche du temps perdu, Tome 1, Du côté de chez Swann. Paris, N.R.F. $52^{\circ}$ édition, pp. 46-49.

s. Noter que l'usage du terme stimulus fait indifféremment référence à un signal simple, ou complexe. 
La première - en fait la plus proche de ce que l'on définit intuitivement par « la mémoire »- relie entre eux les événements ou épisodes. C'est elle qui semble se déplier en relations spatiales dès que Proust a pu retrouver l'ancrage du lit de la tante Léonie et voit ainsi s'enchaîner les différentes vues de Combray dans un ordre tout à fait géographique.

Le second principe de mémoire associe une réponse comportementale à un stimulus, un objet (porter une cigarette à ses lèvres) et sous-tend les réponses automatiques regroupées en savoir faire acquis.

La troisième mémoire associe une réponse émotionnelle à un stimulus (l'émotion qui saisit Proust lorsque l'ensemble de stimulations, madeleine, cuiller, thé tiède, atteint son palais), c'est elle qui est à la base de cette intelligence émotionnelle très à la mode depuis quelques années. Remarquons qu'elle a animé l'étape initiale du processus de reconstruction que Proust s'est efforcé de mener consciemment.

Cette troisième forme de mémoire est très familière, c'est celle justement qui peut nous mettre dans un état d'inconfort à la détection d'une certaine attitude chez un interlocuteur familier sur le point de se fâcher. Au sens psychobiologique, l'émotion est un état fonctionnel à double composante. L'une désigne la manière dont le corps s'adapte, par une posture, un changement d'activité neuro-végétative ou endocrinienne, à une situation (réelle ou imaginaire) qui demande un certain effort (surprise, peur, colère, joie). L'autre face étant la valence attribuée à cette situation et à cette activité adaptative, sa «qualité pour soi», autrement dit, sa pertinence pour l'individu.

Ce cadrage de l'émotion combine les dimensions d'effort adaptatif (l'émotion au sens biologique) avec un processus d'évaluation de la situation (contexte, événement, stimulus particulier) comme l'a montré Buck (1989). Et c'est cette association entre différents composants: soi, la manière d'être qui est une stratégie adaptative, et l'environnement qui représente la mémoire des émotions.

\section{- Animalité de la mémoire épisodique}

L'étude du fonctionnement cérébral s'est organisée selon les structures macroscopiques étudiées, les ventricules tout d'abord, carrefour dans la circulation des humeurs entre le corps et le cerveau, à la Renaissance, puis l'écorce cérébrale qui se prête si facilement à une description topographique. L'extension considérable de cette surface corticale chez les mammifères supérieurs et l'Homme en particulier a alimenté l'idée d'une rupture fonctionnelle entre les structures cérébrales profondes et la surface corticale. Comme si cette dernière faisait le propre de la pensée humaine, alors que la profondeur abritait les restes d'un cerveau ancestral, animal. La définition du lobe limbique par Paul Broca puis du système limbique par Mac Lean (Swanson, 1983), renforce la description d'un humain luttant contre ses émotions et ses instincts avec un néocortex très sophistiqué, mais sans prise sur la profondeur animale de son cerveau. La célèbre phrase de Mac Lean selon laquelle «le néocortex se trouve juché sur le système limbique comme un cavalier sans rênes » (Ganong, 1989) est exemplaire à cet égard. Son cerveau à trois niveaux (1985) a longtemps servi de référence pour tous les modèles fonctionnels, plus symboliques que neurophysiologiques, destinés à convaincre que les difficultés comportementales tenaient à une articulation incertaine entre ces trois 
niveaux. Il ne s'agit pas de faire la critique de Mac Lean, c'est simplement l'occasion de souligner combien l'approche des fonctions cérébrales est contaminée par des biais idéologiques. Elle se prête d'autant plus facilement à des explications symboliques que celles-ci semblent offrir une simplicité qui compense la complexité des explications scientifiques, souvent contradictoires.

Ce découpage a considérablement freiné le développement de la psychobiologie de la mémoire. En effet, la clinique a souligné depuis longtemps l'incidence des lésions du lobe temporal et plus particulièrement de l'hippocampe dans les amnésies et depuis les années 1970, les travaux expérimentaux chez l'animal ont offert des données allant dans ce sens. Comme on peut le comprendre, il était difficilement concevable d'attribuer à une structure incluse dans le système limbique un rôle clé dans la mémoire épisodique humaine, ou d'admettre qu'un animal comme le rat puisse disposer d'une telle forme de mémoire. L'autre difficulté était de concevoir les modalités des interactions entre mémoire et émotions du fait de la conviction d'une domination, chez l'homme, de la raison consciente sur les processus émotionnels (par exemple, Damasio, 1996).

Ainsi il fallut environ 15 ans de recherches convergentes, contradictoires et parallèles, ainsi que toute l'évolution des idées sur la rationalité de la fin du $\mathrm{XX}^{\mathrm{e}}$ siècle pour arriver aux propositions défendues dans l'expérience de McDonald et White. On peut dire que cette évolution repose surtout sur deux lignes de recherches, celles qui ont montré les liens anatomiques forts entre deux régions du système limbique - hippocampe et amygdale - et le néocortex, et celles qui ont permis de formuler des théories complètes pour rendre compte du fonctionnement de l'hippocampe. On peut dire que les données neurophysiologiques ont en quelque sorte désenclavé le système limbique (ou le néocortex?) en mettant en évidence leurs liens étroits. Sur un plan plus conceptuel, la percée provocatrice initiée par O'Keefe et Nadel, en 1978, faisait de l'hippocampe un producteur de carte cognitive - «The hippocampus as a cognitive map». Cette proposition a orienté l'intérêt vers la mémoire de l'espace et les mécanismes sous-jacents. Comme Tolman en 1948 déjà, ils considéraient que l'animal se représente l'espace dans lequel il se déplace en extrayant un ensemble de relations de types topologique entre les différents lieux qu'il est amené à visiter. Comme toute personne dont le chien a été heurté par une voiture peut en faire le constat, l'animal va garder une mémoire du lieu de l'accident, bien plus que du véhicule qui l'a heurté. Comme si l'espace était la matrice de base dans laquelle les événements quotidiens sont fixés, et que leur adresse spatiale était plus importante que leur adresse temporelle.

Cette théorie a résisté à 25 ans d'expériences contradictoires, ce qui témoigne de sa robustesse. Actuellement, on peut dire qu'elle est en butte à deux critiques qui ne l'invalident pas. La première affirme que la mémoire spatiale n'est qu'un cas particulier du codage des relations entre événements par l'hippocampe (Eichenbaum \& al, 1999). La seconde remet en question le caractère unique du codage spatial par l'hippocampe et propose un fonctionnement basé sur deux canaux parallèles (Jacobs \& Schenk, 2003). Aucune de ces deux critiques ne la remettent fondamentalement en question, elles contribuent toutes deux à l'hypothèse que la mémoire spatiale est une forme primaire de la mémoire épisodique. En outre, elles montrent toutes deux comment l'hippocampe respecte une propriété du codage épisodique qui n'est pas forcément spatiale. 


\section{LA DISSOCIATION TRIPLE}

En résumé, les trois freins que nous avions décrits ont été levés progressivement, permettant une compréhension de la mémoire en fonctions abstraites: des relations entre stimuli pour la mémoire épisodique, des relations entre stimulus et réponse pour la mémoire des savoir faire, des relations entre stimuli et réponse émotionnelle pour la troisième (White \& McDonald, 2002). Ces propriétés sont le fait de systèmes cérébraux relativement indépendants, et le consensus presque général d'une mémoire spatiale chez les animaux permet une approche expérimentale rigoureuse.

\section{- Des dissociations simples à la triple dissociation}

La neuropsychologie a très vite montré que des fonctions mentales que l'on pouvait croire unifiées, la compréhension du langage, par exemple, étaient soustendues par le fonctionnement de structures cérébrales voisines et cependant relativement indépendantes. La modularité de l'esprit, soutenue par Fodor (1986) a ainsi été présagée par la multiplicité des modules fonctionnels émergeant de l'étude des cas cliniques. Jusqu'à la caricature de la «région corticale impliquée dans la reconnaissance des Volkswagen vertes » (Roediger \& al, 1990).

Les exemples de dissociations fonctionnelles simples sont innombrables. Ainsi la perte d'une fonction _ (la reconnaissance des visages) lors d'une lésion de la structure cérébrale A et la perte d'une fonction _ (la reconnaissance des outils) lors d'une lésion de la structure complémentaire B offre un exemple de dissociation simple. Mais ces observations ne permettent pas de conclure à l'indépendance fonctionnelle des régions A et $\mathrm{B}$. C'est le principe de la double dissociation, qui peut en faire «la preuve». Selon ce principe, la lésion de A entraîne la perte de la fonction _ et la préservation de _ alors que la lésion de B provoque la perte de la capacité _ sans altération de _, qui est reconnu pour donner une preuve - ou plutôt une indication pour le scientifique prudent - de l'indépendance fonctionnelle des structures cérébrales $\mathrm{A}$ et $\mathrm{B}$ et de leurs fonctions.

De là à des dissociations d'ordre plus élevé, il n'y a qu'un pas, faible pour le logicien habitué à la généralisation. Un pas beaucoup plus grand pour l'expérimentateur qui doit trouver le protocole adéquat, comme on le verra.

\section{- Le protocole expérimental}

L'organisation des mouvements dans l'espace ne requiert pas nécessairement une représentation spatiale à proprement parler. On peut avoir une préférence pour un endroit, sans trop savoir pourquoi, on peut suivre une règle simple consistant à obéir à des flèches ou autres formes de signalisations. C'est cette multiplicité de déterminants à la base des choix à opérer dans un réseau d'allées que White a choisi de dissocier dans un protocole expérimental élégant.

Lorsqu'un rat explore librement une structure faite de huit allées disposées radialement autour d'un espace central, il peut obéir à différentes règles. Si l'extrémité de chaque branche contient une petite quantité de nourriture très savoureuse, une goutte de lait sucré, le rat apprendra spontanément à visiter chaque allée une fois seulement, sans suivre aucun algorithme simple. En fait, il se souvient des 
lieux qu'il a visités précédemment et n'y retourne pas volontiers. On trouve dans la littérature scientifique d'innombrables expériences qui montrent que le rat dispose d'une représentation de la position relative de ces branches (Suzuki \& al, 1980).

Si la présence de la goutte de lait est signalée par une petite lampe allumée à l'extrémité de l'allée, le rat peut apprendre à utiliser ce signal pour faire ses choix. Et s'il a été confiné dans deux des branches de manière répétée et que seule une des deux branches contenait de la nourriture, il tendra à rester plus longtemps dans la branche qui contenait de la nourriture que dans celle qui était vide lorsque le choix lui sera donné de visiter librement l'une et l'autre. Aucune branche n'étant appâtée dans ce test, seule la valence positive attribuée à l'une des deux branches sera à l'origine d'une préférence facile à objectiver'.

Conformément aux attentes engendrées par la théorie d'O'Keefe et Nadel (1978), les animaux avec lésion de l'hippocampe sont devenus incapables de se souvenir des branches qu'ils ont visitées et font de nombreuses répétitions avant d'achever leur parcours. Toutefois, ils sont très efficaces quand ils peuvent suivre l'information de la petite lampe, et ils expriment une préférence marquée pour l'allée dans laquelle ils avaient trouvé de la nourriture dans la phase de confinement. En somme ces rats ne savent plus s'orienter, mais sont capables de suivre des informations locales ou de marquer une préférence pour une branche particulière.

Les lésions du néostriatum (une partie des ganglions de la base, structures impliquées dans la mise en œuvre de réponses à des stimuli simples) n'interfèrent ni avec la capacité de patrouiller efficacement dans le labyrinthe, ni avec la manifestation d'une préférence pour une de deux branches. Les rats porteurs de lésion du striatum sont incapables de développer la stratégie simple consistant à faire leurs choix selon l'état de la petite lumière placée à l'extrémité de certaines branches.

Enfin les lésions de l'amygdale préviennent l'expression d'une préférence pour la branche qui était garnie de nourriture mais n'interfèrent ni avec une organisation efficace des visites libres, ni avec la capacité de suivre la règle associant la lumière avec la présence de nourriture.

\section{APRÈS LA PREUVE}

Le principe de la triple dissociation est donc satisfait. Cette expérience offre une manière de preuve du parallélisme, donc de l'indépendance de trois formes de mémoire chez l'animal. Mais ce travail n'apporte pas directement d'information nouvelle. Il marque cependant une articulation dans le courant de la recherche en permettant de formuler de nouvelles questions et de nouvelles hypothèses de travail. En effet, si ces capacités de mémoire peuvent s'exprimer indépendamment les unes des autres, la question de leurs interactions chez un individu intact peut enfin être posée en référence à des modes de l'activité cérébrale.

Il va de soi qu'il ne reste pas de traces odorantes permettant de faire un choix correct. 
Le développement d'habitudes se fait à partir de la répétition de décisions qui peuvent être volontaires au départ, et qui se transforment progressivement en des réponses rapides au signal le plus simple et le moins variable qui précédait systématiquement ce comportement. L'automatisme échappe alors au choix volontaire. Ce qui, pour proposer une réduction brutale, pourrait impliquer un «passage du contrôle du comportement de l'hippocampe au striatum», comme si ces deux structures pilotaient des stratégies comportementales différentes. Une hypothèse que White a soutenue par plusieurs expériences intéressantes reposant sur le principe de la double dissociation (Devan et White, 1999). La question des interactions entre systèmes de mémoire est également fondamentale dans le domaine des relations entre ce que l'on peut appeler les cognitions chaudes et froides, dans le cas qui nous occupe, la mémoire des associations entre une émotion et un environnement, dépendant de l'amygdale, et la mémoire des relations spatiales entre différents lieux inscrits dans un environnement, dépendant de l'hippocampe.

La question des interactions entre mémoire et émotion est ancienne. Le modèle proposé par Metcalfe et Jacobs (1999) évoque un couplage fonctionnel qui pourrait être synergiste pour les émotions d'intensité faible à moyenne, puis antagonistes quand l'intensité dépasse un certain niveau. Une manière de rendre compte du fait bien connu que l'on est facilement désorienté si l'on s'affole. Mais la question du mode d'organisation de la mémoire dans ces circonstances est de celle qui préoccupe le psychiatre lorsqu'il traite un patient qui ne parvient pas à organiser le souvenir qu'il garde d'un épisode traumatisant, comme si l'événement était enfermé dans une sorte de bulle déconnectée de son ancrage spatiotemporel.

On pourrait dire alors que l'évolution des connaissances marquée par cette preuve est susceptible de permettre des transpositions des connaissances en neurobiologie vers des disciplines à visées thérapeutiques, une des finalités de la neurobiologie.

\section{CONCLUSION}

La preuve, ce moyen que l'on voudrait indiscutable d'établir une vérité, représente surtout l'achèvement d'une évolution vers un contexte consensuel en dépit des lacunes dans les connaissances et des biais conceptuels. En somme tout se passe comme s'il fallait convaincre bien avant de fournir une preuve, convaincre de ce que cette preuve en est bien une.

Cette réflexion sur la démarche expérimentale, conduite «à chaud», comme on fait de l'histoire contemporaine, illustre semble-t-il les bénéfices de l'interdisciplinarité. Contrairement à ce qu'affirment parfois les sociologues, les neurobiologistes disposent eux-mêmes des éléments nécessaires pour prendre une certaine distance par rapport à leur démarche. Il faut cependant, et c'est une difficulté récurrente, parvenir à transposer une la question posée par les sciences humaines aux sciences biologiques. Cette transposition oblige à des simplifications qui ne doivent pas devenir triviales pour les biologistes, il faut aussi qu'ils soient à l'écoute des questions posées par les chercheurs en sciences humaines. L'idée d'un langage commun supposerait que celui-ci soit universel, au moins dans le sous-groupe social de recherche. Le problème tient au fait que la psychobiologie étant en partie une science humaine, elle doit - en fait elle veut - utiliser des 
concepts propres à cette discipline (mémoire, émotion, attention etc.). Mais elle doit se les approprier au préalable. D'où le fait que les ambiguïtés du langage d'un champ de recherche s'amplifient encore lors de cette transposition.

\section{Université de Lausanne}

\section{BIBLIOGRAPHIE}

Buck, R. (1989) Subjective, expressive, and peripheral bodily components of emotion. In Wagner, H, Manstead, A (Edit), Handbook of social psychophysiology, John Wiley, Chichester, 199-222.

Churchland, P.S. (1988). The significance of neuroscience for philosophy. Trends in Neurosciences. 11, 304-307.

Damasio, A. (1994). L'erreur de Descartes. Editions. O. Jacob, Paris

Devan, B.D. \& White, N.M.(1999). Parallel information processing in the dorsal striatum: relation to hippocampal function. Journal of Neuroscience. 19, 2789-98.

Eichenbaum, H., Dudchenko, P., Wood, E., Shapiro, M., \& Tanila, H. (1999). The hippocampus, memory, and place cells: Is it spatial memory or a memory space? Neuron, 23, 209-226.

Fodor, J.A. (1986). La modularité de l' esprit. Paris, Editions de Minuit.

Gallese, V. \& Goldman, A. (1998). Mirror neurons and the simulation theory of mind reading. Trends in Cognitive Sciences, 2, 493-501

Ganong, W.F. (1989) Review of medical physiology. Appleton \& Lange, Prentice Hall.

Jacobs, L.F. \& Schenk, F. (2003). Unpacking the cognitive map: the parallel map theory of hippocampal function. Psychological Review, 110, 285-315.

MacLean, P.D. (1985). Evolutionary psychiatry and the triune brain. Psychological Medicine, 15, 21921.

McDonald, R.J. \& White, N.M. (1993). A triple dissociation of memory systems: hippocampus, amygdala, and dorsal striatum. Behavioral Neuroscience, 107, 3-22.

Metcalfe, J. \& Jacobs, W.J. (1998) Emotional memory: The effects of stress on «cool» and «hot» memory systems. Medin, Douglas L. (Ed).. The psychology of learning and motivation : Advances in research and theory, Vol. 38. (pp. 187-222), 306pp.

Milner, B. (1965). Memory disturbance after bilateral hippocampal lesions. In: P. Milner \& S. Glickman (Eds): Cognitive processes and the brain. Princeton, N.J.: Van Nostrand.

O'Keefe, J. \& Nadel, L. (1998). The hippocampus as a cognitive map. Oxford University Press.

Proust, M. (1919). A la recherche du temps perdu, Tome 1, Du côté de chez Swann. Paris, N.R.F.

Roediger, H.1., Rajaram, S., \& Srinivas, K. (1990). Specifying criteria for postulating memory systems. Annals of the New York Academy of Sciences, 608, 572-595.

Schenk, F. (1992) La mémoire chez l'animal: évolution récente de quelques concepts utilisés en psychophysiologie. L'Année Psychologique, 92, 233-261.

Squire, L.R. \& Zola-Morgan S. (1988) Memory: brain systems and behavior. Trends in Neurosciences, 11, 170-5.

Suzuki, S., Augerinos, G., \& Black, A.H. (1980). Stimulus control of spatial behavior on the eight-arm maze in rats. Learning and Motivation, 11, 1-18.

Swanson, L.W. (1983) The hippocampus and the concept of the limbic system. In Seifert, CW (Edit). Neurobiology of the hippocampus, 3-19, New York, Academic Press.

Tolman, E.C. (1948). Cognitive maps in rats and men. Psychological Review, 55, 189-208.

White, N.M. \& McDonald, R.J. (2002). Multiple parallel memory systems in the brain of the rat. Neurobiology of Learning \& Memory, 77, 125-84. 